\title{
Linx
}

Revue des linguistes de l'université Paris X Nanterre

$81 \mid 2020$

Enseignement des langues : la grammaire et la linguistique sont-elles solubles dans la pédagogie de projet ?

\section{La conceptualisation grammaticale comme objet du projet en cours d'anglais langue étrangère : bilan d'une expérience en classe de seconde}

Grammatical conceptualisation as a project for the EFL class; taking stock of an experiment in high school

Agnès Leroux et Virginie Plessis

\section{(2) OpenEdition}

Journals

Édition électronique

URL : http://journals.openedition.org/linx/7253

DOI : $10.4000 / \operatorname{linx} .7253$

ISSN : 2118-9692

Éditeur

Presses universitaires de Paris Nanterre

Édition imprimée

Date de publication : 15 décembre 2020

ISSN : 0246-8743

Référence électronique

Agnès Leroux et Virginie Plessis, « La conceptualisation grammaticale comme objet du projet en cours d'anglais langue étrangère : bilan d'une expérience en classe de seconde », Linx [En ligne], 81 | 2020, mis en ligne le 15 décembre 2020, consulté le 29 janvier 2021. URL : http://

journals.openedition.org/linx/7253; DOI : https://doi.org/10.4000/linx.7253

Ce document a été généré automatiquement le 29 janvier 2021.

Département de Sciences du langage, Université Paris Ouest 


\section{La conceptualisation grammaticale comme objet du projet en cours d'anglais langue étrangère : bilan d'une expérience en classe de seconde}

Grammatical conceptualisation as a project for the EFL class; taking stock of an experiment in high school

\section{Agnès Leroux et Virginie Plessis}

\section{Introduction}

Cet article rend compte d'une expérience de type « recherche-action » menée en classe de seconde sur l'année scolaire 2017-2018, dans un lycée de Fontainebleau. L'objectif de l'enseignante était de parvenir à travailler l'objectif grammatical dans le cadre de l'approche à visée actionnelle, conseillée par le Cadre Européen Commun de référence pour l'Enseignement des Langues (désormais CECRL) et auquel s'adossent les Instructions Officielles françaises. Plus précisément, suite à une formation en M2 recherche en option linguistique à l'Université, cette enseignante voulait aborder la conceptualisation $\mathrm{du}$ rapport entre temps chronologique et temps et aspect grammaticaux en anglais, en classe de seconde. Sans perdre de vue la compétence de communication ni l'ancrage culturel de ses séquences, elle pensait proposer une réflexion grammaticale à ses élèves de seconde (première année de secondaire supérieur) pour les amener progressivement à comprendre ce qui se joue entre le français et l'anglais, respectivement leur langue de scolarité (désormais LS) et leur première langue vivante étrangère (désormais LVE). 
2 Ce travail a pris la forme d'un projet de classe dont la production finale s'est révélée très efficace pour la progression linguistique et langagière des élèves et leur confiance personnelle en cours de LVE.

3 Plus qu'un compte-rendu d'expérience menée sur une classe, il s'agit ici d'analyser les résultats obtenus au fur et à mesure de l'année, tant en termes quantitatifs que qualitatifs, et de questionner la place de l'objectif linguistique dans la construction du projet de séquence. En d'autres termes, nous voudrions montrer ici que le terrain d'entente entre approche à visée actionnelle et objectif linguistique peut ne pas se résumer à une simple condition de construction du sens mais constituer la matière du projet lui-même.

4 Nous prendrons comme point de départ le cadre législatif de l'enseignement des langues étrangères en milieu institutionnel afin de mettre en évidence le paradoxe de la relation entre l'approche préconisée et les contraintes inhérentes à l'enseignementapprentissage d'une langue en milieu institutionnel. Nous présenterons ensuite l'expérience menée en classe d'anglais langue étrangère (désormais ALE) ainsi que son analyse. Cela nous permettra de montrer que le chemin choisi par l'enseignante respecte le cadre imposé par l'institution en même temps qu'elle remet au centre de l'apprentissage la relation entre la LVE et l'apprenant.

\section{Analyse de l'approche à visée actionnelle en cours de LVE}

5 Afin d'harmoniser l'enseignement des langues sur l'Europe, dès 1977 le Conseil de l'Europe a établi un ensemble de règles qu'il est souhaitable que les différents états européens fassent appliquer dans les établissements d'enseignement primaire et secondaire.

\subsection{Définition de l'approche à visée actionnelle selon le Cadre Européen Commun de référence pour les Langues (CECRL, 2003)}

6 Le CECRL, préconise de privilégier à la fois la dimension communicationnelle et le « lien social» créé par la langue, elle-même envisagée comme un outil d'action sur l'environnement à la disposition de l'apprenant :

La perspective privilégiée ici est, très généralement aussi, de type actionnel en ce qu'elle considère avant tout l'usager et l'apprenant d'une langue comme des acteurs sociaux ayant à accomplir des tâches (qui ne sont pas seulement langagières) dans des circonstances et un environnement donnés, à l'intérieur d'un domaine d'action particulier. Il y a " tâche " dans la mesure où l'action est le fait d'un (ou de plusieurs) sujet(s) qui y mobilise(nt) stratégiquement les compétences dont il(s) dispose(nt) en vue de parvenir à un résultat déterminé (CECRL, 2003).

7 Cette définition est suffisamment large pour laisser une grande liberté didactique et pédagogique aux enseignants de LVE, mais elle donne clairement la définition d'une tâche à visée actionnelle en fonction de cinq critères essentiels :

- l'action doit être le fait d'un ou de plusieurs sujets

- qui utilisent des compétences

- pour élaborer une stratégie

- en vue de parvenir à un résultat 
- déterminé à l'avance.

8 Le paradoxe du cours de langue naît cependant de ce qu'il est explicitement indiqué que les tâches ne sont pas «seulement langagières ", que les compétences à mettre en œuvre peuvent être autres que communicationnelles et que le résultat attendu peut sortir du domaine strict de l'enseignement des langues. Il est donc raisonnable d'en déduire qu'il peut s'agir d'une réalisation engageant des compétences linguistiques et langagières, à destination de groupes intérieurs ou extérieurs à la classe. Dès lors l'enseignant de langue est en droit de poser la question de l'objet de son enseignement et de l'évaluation : évalue-t-on la forme que prend la langue ou le résultat de l'action commune d'un groupe d'élèves dont la réussite peut reposer sur bien d'autres compétences que le linguistique et le langagier?

\subsection{Expression de l'approche à visée actionnelle dans les Instructions Officielles (I.O.) françaises}

9 Dans les I.O. françaises de 2019 pour l'enseignement des langues, il apparaît que l'approche à visée actionnelle est envisagée comme l'enseignement de la langue à partir de scénarios de classe, scénarios pédagogiques :

En classe, l'enseignant privilégie la mise en place de projets autour de scénarios pédagogiques qui articulent les entraînements, les contextualisant, et aboutissent progressivement à des productions écrites ou orales (...). L'enseignant s'attache à mettre l'élève en action et l'encourage à prendre des initiatives à travers les activités proposées. Certains scénarios peuvent faire l'objet d'une diffusion dans le cadre du lycée par l'intermédiaire du journal et/ou de la radio, du Centre de connaissance et de culture $(3 \mathrm{C}$, anciennement Centre de Documentation et d'information CDI), de l'Environnement numérique de travail (ENT), du site internet de l'établissement. (I.O. parues au Bulletin Officiel en janvier 2019)

10 La grammaire n'est pas évincée pour autant puisqu'il existe une partie intitulée 'correction grammaticale' dans laquelle l'étude de la langue elle-même est présentée comme garante d'un apprentissage réussi :

Dans la perspective de l'acquisition d'une langue vivante, l'étude de la grammaire est l'un des outils permettant de complexifier et de perfectionner le maniement de la langue et d'enrichir la réflexion. C'est en progressant dans la compréhension des mécanismes de la langue que l'élève devient de plus en plus autonome. A partir des documents abordés en cours, l'élève est conduit à découvrir les règles d'usage de la langue, à identifier les points de syntaxe ou de morphologie constitutifs du document, afin de se les approprier. Il est ensuite entraîné à les remobiliser de manière fréquente. Une meilleure maîtrise de la grammaire donne sens et cohérence aux énoncés produits. (B.O. de janvier 2019, pour l'enseignement des LVE en classe de seconde)

11 La grammaire est un objectif à part entière, au service de la construction du sens à des fins de communication, et sert un objectif conceptuel puisque sa contribution à l'enrichissement de la réflexion est explicitement mentionnée.

12 Cette réflexion doit cependant toujours s'exercer dans le cadre des scénarios destinés à contextualiser l'apprentissage. Elle reste donc un outil de second plan. 


\subsection{Le point de vue de quelques chercheurs en enseignement- apprentissage des LVE} caractéristiques linguistiques de la langue étudiée, il faut que l'organisation des tâches suive l'organisation structurelle de la langue (focus on forms). Il en résulte une planification de l'organisation des activités de classe en fonction de la structure des faits de langue que l'apprenant doit acquérir. Ellis envisage donc l'acquisition d'une LVE comme un processus lors duquel les structures de la langue seraient, à long terme et de façon implicite, automatisées et reliées à certaines situations de communication. Par exemple, un jeu de mime en anglais en cycle 3 ou début de cycle 4, associé à la forme be+Ving permettrait à l'apprenant de faire le lien entre forme et sens, et à long terme de catégoriser les situations de communication qui requièrent l'emploi de cette structure.

19 Tout en n'étant pas d'accord avec cette conception de l'apprentissage d'une langue, qui relève d'une méthode inductive implicite, Chini reconnaît que la méthodologie du cours induite par l'approche à visée actionnelle en regard du nombre d'heures dévolues au cours de langue dans l'emploi du temps des élèves, ne laisse que peu de temps à l'apprentissage par réflexion sur le lien entre structure et construction du sens (dynamique psycholinguistique). C'est donc de fait l'activité langagière en termes de 
performance et le produit de la tâche finale qui sont favorisés. Faut-il pour autant considérer que la solution suggérée par Ellis soit l'unique viable?

\subsection{Particularité des conditions d'apprentissage d'une LE en milieu institutionnel}

Cette divergence sur les procédés à mettre en œuvre pour l'apprentissage effectif d'une LVE prend racine dans l'essence même de la relation enseignement-apprentissage en milieu institutionnel.

En effet, enseignant et apprenants partagent une même langue de scolarité et auraient par conséquent peu de raisons de communiquer dans une autre langue, s'ils ont vraiment quelque chose à se dire, et le sujet essentiel d'une potentielle discussion est l'objet d'étude, la LVE. Celle-ci doit néanmoins devenir en plus d'objet d'étude outil de communication au sein de la classe, ciment social du groupe classe, et support de l'évaluation. Ce rapport à l'objet d'étude et d'apprentissage est particulier à l'enseignement des LVE en milieu institutionnel, aucune autre discipline ne fonctionne ainsi.

Dans les I.O. françaises de 2015, la réflexion sur la langue n'était pas exclue :

C'est l'exposition régulière et quotidienne à la langue qui favorise les progrès des élèves; son utilisation en contexte donne du sens aux acquisitions. Un début de réflexion sur le fonctionnement de la langue permet aux élèves d'acquérir une certaine autonomie dans la réception et dans la production et renforce la maitrise du langage. Indissociable de l'apprentissage de la langue [...]. (I.O françaises de novembre 2015, cycle des approfondissements)

Ils offraient une entière liberté aux enseignants sur les moyens d'atteindre les objectifs, puisqu'il était stipulé que «ils laissent [les enseignants] apprécier comment atteindre au mieux les objectifs des programmes en fonction des situations réelles qu'ils rencontrent dans l'exercice quotidien de leur profession ».

Il n'en reste pas moins que si l'on favorise "l'exposition régulière et quotidienne à la langue ", et que l'objectif de chaque séquence est la construction d'un projet commun à la classe, à raison de trois heures par semaine, il ne reste que peu de temps pour la réflexion linguistique elle-même et pour l'acquisition langagière telle qu'envisagée par Ellis.

Malgré tout, la difficulté des apprenants à construire le sens en expression et compréhension avec les structures linguistiques de la LVE nous est apparue comme une 'situation réelle rencontrée dans l'exercice quotidien de la profession' d'enseignant d'anglais, et nous sommes partie de ce principe pour prendre l'entrée linguistique comme support de projet. Nous allons montrer qu'il est possible d'adopter une démarche qui permette, malgré une entrée linguistique, de ne pas aller à l'encontre de l'ancrage culturel du cours de LVE.

\section{Description de l'expérience}

L'expérience présentée ici prend pour support une situation réelle rencontrée dans l'exercice quotidien de la profession d'enseignant de langue : le défaut de maîtrise du système aspecto-temporel de l'anglais par des élèves entrant en seconde. Nous en 
donnons un exemple ci-dessous, à partir de l'évaluation diagnostique effectuée par l'enseignante en novembre, et dont nous reparlerons en partie 3 de cet article :

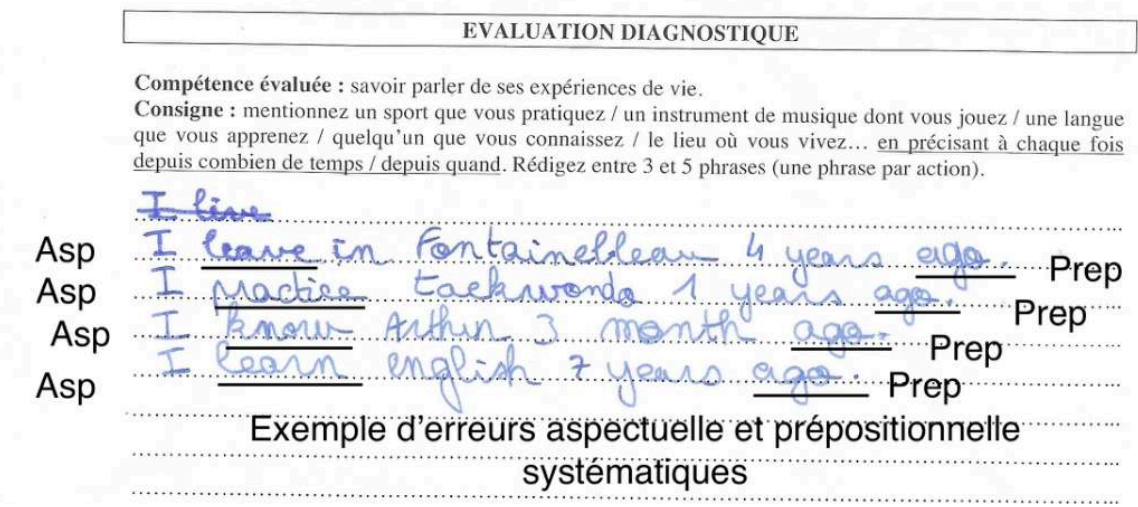

Évaluation 1.

L'enjeu de la référence au temps en anglais pour des apprenants francophones, est de taille puisque les deux systèmes aspectuo-temporels sont fondamentalement différents malgré quelques similitudes de surface :

En français, nous avons plusieurs modes, à l'intérieur desquels se développent plusieurs formes de temps grammatical pour référer au temps: présent de l'indicatif, présent du subjonctif, passé-composé à l'indicatif, passé simple de l'indicatif ou du subjonctif, futur de l'indicatif, etc. Chacune de ces formes entretient un rapport particulier avec le temps chronologique, et avec le réel ou l'irréel.

En anglais, il n'existe que deux temps et deux aspects, les modes étant presque toujours représentés par des auxiliaires modaux. Le prétérit, unique temps du passé, réfère au révolu et à l'irréel, tout ce qui n'est pas en rapport direct avec le moment de discours, et le présent réfère à tout ce qui peut inclure le moment de discours. Les deux aspects en Have-en et Be-ing, permettent de situer les événements par rapport au moment origine du discours.

Les erreurs des apprenants francophones, de par leur récurrence, relèvent certainement de ce que Tardif (1994) pointe comme une surgénéralisation suite à transfert erroné à partir de la LS. Elles nécessitent un traitement particulier. Il semblait intéressant de tenter une focalisation plus explicite sur ce phénomène linguistique, afin d'amener les apprenants à déconstruire leurs apprentissages antérieurs, sources soit de surgénéralisation soit d'hypothèses erronées, tout en leur offrant l'opportunité de construire collectivement une activité de réflexion sur la langue afin d'expliciter leur propre interprétation métalinguistique du rapport entre LS et LVE.

Cet objectif conceptuel n'a pas amené l'enseignante à écarter le but ultime de la pédagogie de projet. En effet, il s'agissait de concevoir collectivement un dispositif métalinguistique issu de la réflexion des élèves. Ce dispositif devait avoir pour objectif d'aider les élèves à construire le sens des déterminations relatives au temps grammatical et à l'aspect sur le verbe en anglais. La description de l'expérience montre qu'il y a eu négociation sur le sens d'un document authentique, article de presse relatant l'expérience d'un survivant aux attentats du 11 septembre. Cette négociation a été le point de départ d'un projet pédagogique dont l'issue matérielle et tangible a pu ensuite être exploitée à diverses reprises lors d'interventions de remédiation mais aussi lors de séances d'expression orale. Si ce projet est perfectible, pour les raisons qui seront exposées en troisième partie, il n'en reste pas moins un exemple d'exploitation 
du potentiel didactique de la linguistique et de la grammaire en cours de LVE dans l'enseignement secondaire.

\subsection{Sujets observés}

Le niveau retenu pour cette expérience est la classe de Seconde, cinquième année de l'enseignement secondaire, car le système de référence au temps chronologique étant initialement introduit en troisième année, il est encore relativement frais dans les esprits des élèves. Par conséquent, les représentations erronées sont sans doute moins ancrées, ou fixées, qu'à un niveau supérieur. Mais surtout, le volume horaire consacré au cours d'anglais, plus important à ce niveau (trois heures hebdomadaires contre deux-et-demi en Première et deux en Terminale), et l'absence de contraintes liées à des examens terminaux rendent la marge de manœuvre pour mener l'expérimentation plus favorable qu'à un niveau sanctionné par un examen.

31 L'expérience a été conduite simultanément sur deux groupes, afin d'établir un protocole expérimental satisfaisant composé d'un groupe test et d'un groupe témoin.

32 Le groupe test a bénéficié de la démarche expérimentale à partir de leur activité réflexive instantanée sur la langue, aussi appelée activité épilinguistique.

Avec le groupe témoin, l'expression de la durée repérée a été abordée suivant l'approche métalinguistique proposée dans le précis de grammaire de leur manuel (New Bridges Seconde, Paris, Nathan).

\subsection{Modalités d'observation}

L'expérience complète, construction collective du dispositif métalinguistique et moments d'évaluation, s'est déroulée sur une année scolaire, de septembre à mai.

Afin d'évaluer le plus justement et le plus scientifiquement possible l'efficacité de la démarche expérimentale, des évaluations régulières, communes aux 2 groupes, ont été effectuées, à quatre moments numérotés de $\mathrm{T}_{0}$ à $\mathrm{T}_{3}$ :

- $\mathrm{T}_{0}$ : une évaluation diagnostique orale mi-septembre

- $\mathrm{T}_{1}$ : une évaluation écrite, mi-novembre (exposée en partie 3)

- $\mathrm{T}_{2}$ : une évaluation écrite post-remédiation en février, de consigne similaire à

l'évaluation diagnostique initiale afin de pouvoir quantifier la progression

- $\mathrm{T}_{3}$ : enfin, une évaluation écrite, bilan ou post test en mai, dont le but était

d'estimer le taux de fixation des acquis.

\subsection{Déroulement de la phase expérimentale}

Tableau 1.

\begin{tabular}{|l|l|l|}
\hline Quoi ? & Comment ? \\
\hline 1. & $\begin{array}{l}\text { Hypothèses sur la construction du sens } \\
\text { d'un texte par une représentation visuelle }\end{array}$ & $\begin{array}{l}\text { Élaboration individuelle d'une représentation } \\
\text { graphique de la chronologie des événements } \\
\text { présentés par le texte }\end{array}$ \\
\hline
\end{tabular}




\begin{tabular}{|l|l|l|}
\hline 2. & $\begin{array}{l}\text { Construction d'un discours naif, initiation } \\
\text { du processus de réflexion collective }\end{array}$ & $\begin{array}{l}\text { Présentation orale de chaque production par son } \\
\text { auteur (1 séance) }\end{array}$ \\
\hline 3. & $\begin{array}{l}\text { Réflexion sur l'élaboration d'un outil } \\
\text { métalinguistique }\end{array}$ & Négociation sur un dispositif commun (1 séance) \\
\hline 4. & $\begin{array}{l}\text { Expression, remédiation et repérages } \\
\text { dans le temps }\end{array}$ & $\begin{array}{l}\text { Manipulation fréquente de l'outil graphique et } \\
\text { modifications }\end{array}$ \\
\hline
\end{tabular}

L'activité de construction du dispositif métalinguistique s'est déroulée en quatre grandes étapes, partant de la construction du sens d'un texte authentique sous forme de représentation graphique, pour aboutir à l'expression en semi-autonomie à l'aide du dispositif élaboré collectivement.

L'objectif était d'offrir aux élèves un support de conscientisation qu'ils pourraient ensuite réinvestir de façon collective ou individuelle, selon les besoins. L'enseignante a respecté les différents stades possibles de conscientisation définis par Bailly (1984). La conscientisation commence dès l'entrée en cours de langue : les consignes, par exemple participent de la méta-situation d'apprentissage. Il s'agit d'un stade verbal de conscientisation. La phase de verbalisation permet de passer à un stade semi-verbal pour construire avec les élèves une représentation physique du travail cognitif effectué en cours de langue : tentatives de manipulation de structures, de construction de graphiques, qui leur permettent d'objectiver puis de confronter leurs hypothèses sur la langue à celles des autres élèves, phase 1 et 2 du tableau 1 . Une fois le semi-verbal exploré, il est possible de mettre en œuvre un schéma totalement non verbal, utilisé comme représentation métalinguistique. Il peut s'agir, par exemple, d'un graphique ou de gestes, dont la représentation négociée garantit le statut universel au sein de la classe, phase 3 du tableau 1.

\subsubsection{Première étape, représentation visuelle : construction du sens du texte}

L'enseignante a commencé par un travail sur la référence au temps, à partir de l'étude d'un texte. Il s'agissait du témoignage d'un survivant à l'effondrement des Twin Towers paru dans le journal The Guardian, accompagné d'un questionnaire d'aide à la compréhension. L'une des questions posées invitait l'élève à rétablir la chronologie du déroulement de la journée de cet homme, en numérotant de 1 à 12 les événements listés dans le désordre. Un travail de recherche personnel sur ce moment de l'histoire américaine permettait ensuite aux élèves d'inscrire plus exactement cette expérience individuelle dans la chronologie des divers événements des attentats, en ajoutant des horaires en regard des actions.

Une fois le texte étudié et la chronologie rétablie dans le questionnaire, les élèves ont eu pour consigne de proposer une représentation graphique du déroulement de cette journée du témoin interrogé. La consigne a été donnée en français afin de limiter les incompréhensions, et volontairement non explicitée davantage, malgré les questions des élèves (Il faut faire un dessin, c'est ça? Qu'est-ce qu'il faut faire exactement?), afin de ne pas influencer leurs productions. Ce mode opératoire fut également sélectionné pour tenter d'accéder à un mode de pensée individuel, difficilement objectivable (Trévise, 1994 : 172). 
Les vingt productions obtenues ont été collectées, et étudiées par l'enseignante, qui a elle-même identifié deux grandes catégories qui seront présentées ici par la suite. Chaque production a été analysée et commentée oralement par les élèves en classe, indépendamment de ces deux catégories: justification du type de représentation par l'élève producteur, puis questions ou observations par ses camarades.

\subsubsection{Deuxième étape, construction d'un discours naïf : représentation personnelle de la chronologie}

Les dessins obtenus sont classés ci-dessous en fonction du niveau de conceptualisation $\mathrm{du}$ lien entre désinence verbale et temps chronologique de chaque producteur, du plus figuratif au plus conceptuel, comme illustré ci-après.

Les dessins reproduits ici témoignent d'une forte prépondérance du format horizontal (16 productions sur 20) et de la ligne ou frise chronologique (14 productions sur 20), fléchée. Le sens de lecture gauche-droite est systématique. L'homogénéité culturelle du groupe et le caractère familier de la frise chronologique, que les élèves connaissent et manipulent depuis l'école primaire en Histoire, Science et Vie de la Terre ou Histoire de l'Art, sont certainement la cause de ce résultat.

43 Il s'est avéré néanmoins très intéressant de constater que tous ne fonctionnent pas sur le même modèle et peuvent s'inspirer d'autres sources, moins scolaires que la frise (BD, mangas, story boards, etc.).

C'est cependant cette dernière catégorie, avec six exemplaires sur vingt, qui est le moins représentée. En voici un exemplaire :

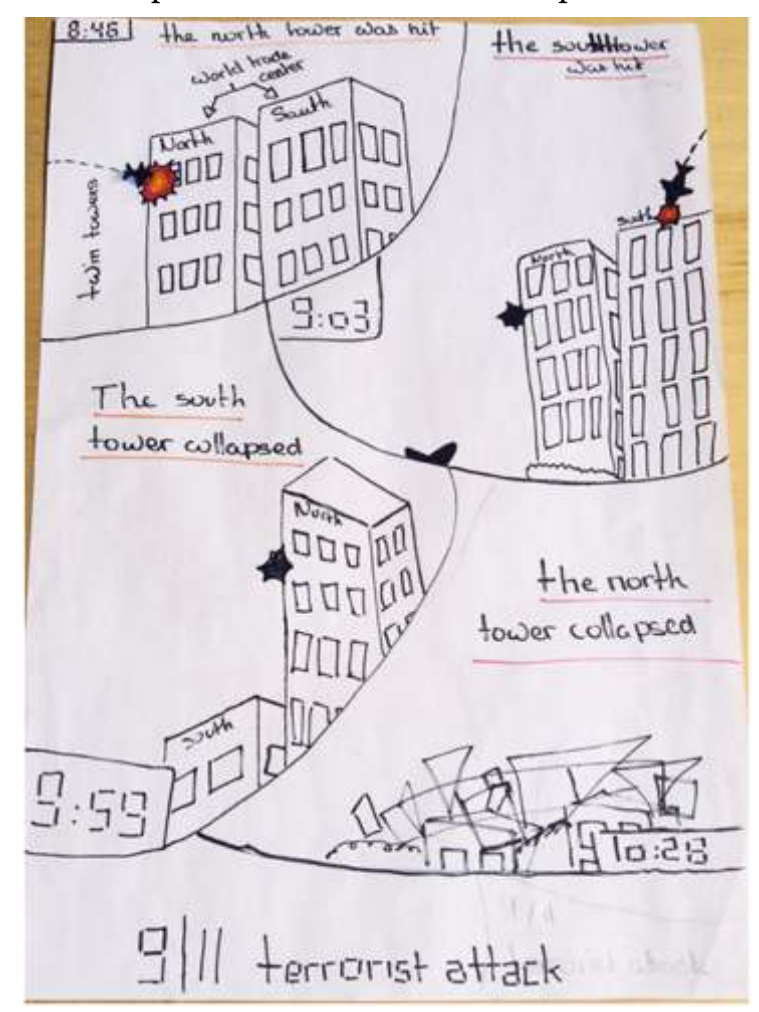

Représentation 1.

L'enchaînement chronologique est représenté par la juxtaposition, suivant une convention d'orientation gauche-droite puis haut-bas, des vignettes contenant chacune 
une étape essentielle des événements. Cette représentation est figurative et non généralisante. Elle permet de comprendre le texte mais ne peut être utilisée pour une exploitation du sens construit par les désinences verbales en anglais pour tout autre texte.

La catégorie majoritairement représentée, avec quatorze productions sur vingt était la représentation type « ligne du temps », toutes orientées sur l'axe horizontal, la plupart fléchées à droite, donc orientées gauche-droite.

Certaines sont encore très proches d'une représentation figurative, comme celle-ci :

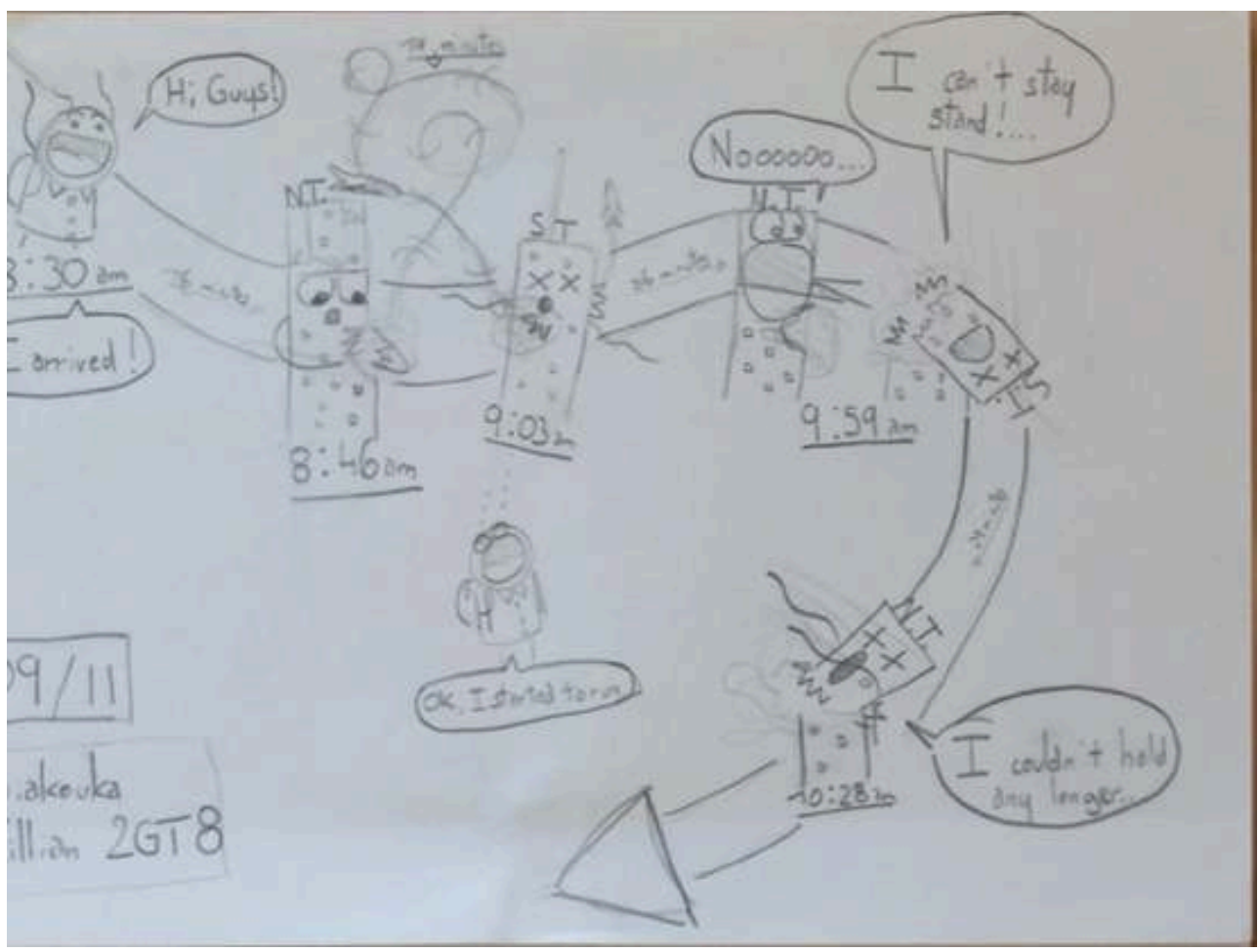

Représentation 2.

Sur cette représentation, représentation 2, si l'élève a détaché les événements les uns des autres pour les situer sur une flèche métaphorique en forme de piste de ski, le mélange de discours direct, introduit par l'élève, au présent (I can't stay stand!) et au passé (I couldn't hold any longer), ainsi que l'utilisation de la première personne du singulier, témoignent d'une absence de conceptualisation de la relation entre temps chronologique et temps grammatical.

Certaines productions, moins figuratives, la succession chronologique étant représentée par une flèche simple, suggèrent un début de conceptualisation du temps par leur producteur : 


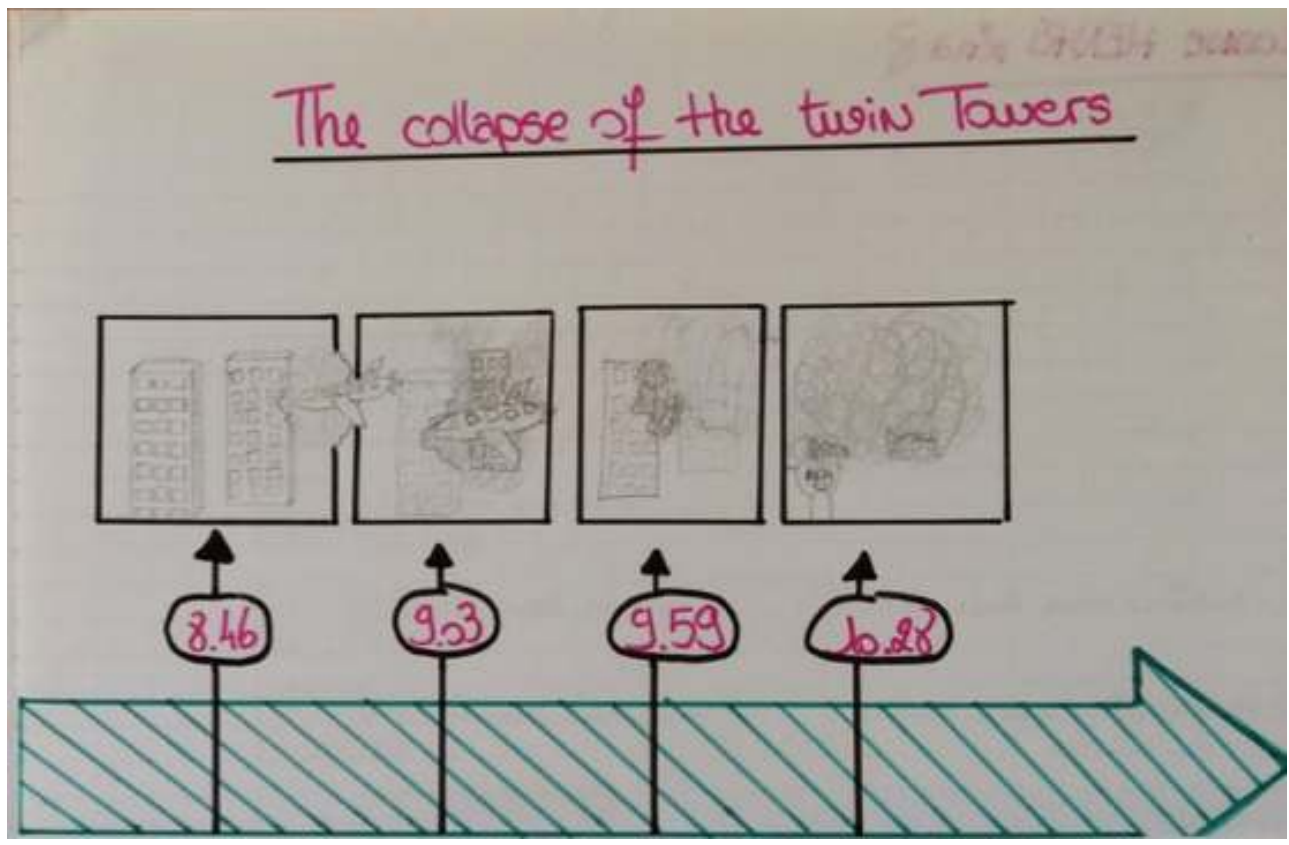

Représentation 3.

Mais le caractère abstrait de la ligne du temps n'efface pas le côté très figuratif des dessins.

La ligne du temps sur la représentation 4, introduit plusieurs éléments supplémentaires : la relation au présent, construite à la fois par le présent simple et symbolisée par un point postérieur aux événements situé sur 11 a.m., le doublage des dessins par des phrases à la troisième personne du singulier, et le rapport au passé construit par l'expression au prétérit :

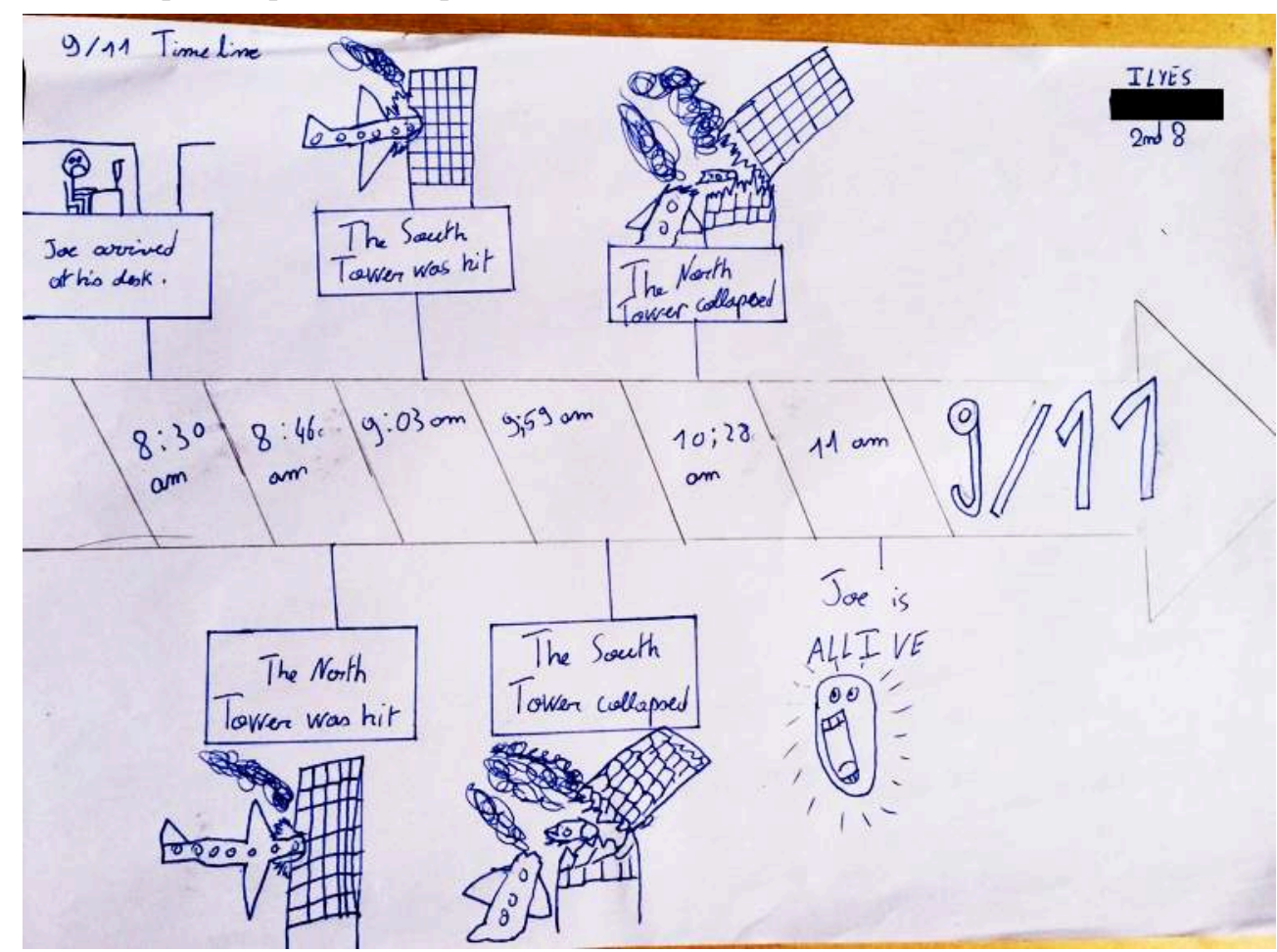

Représentation 4. 


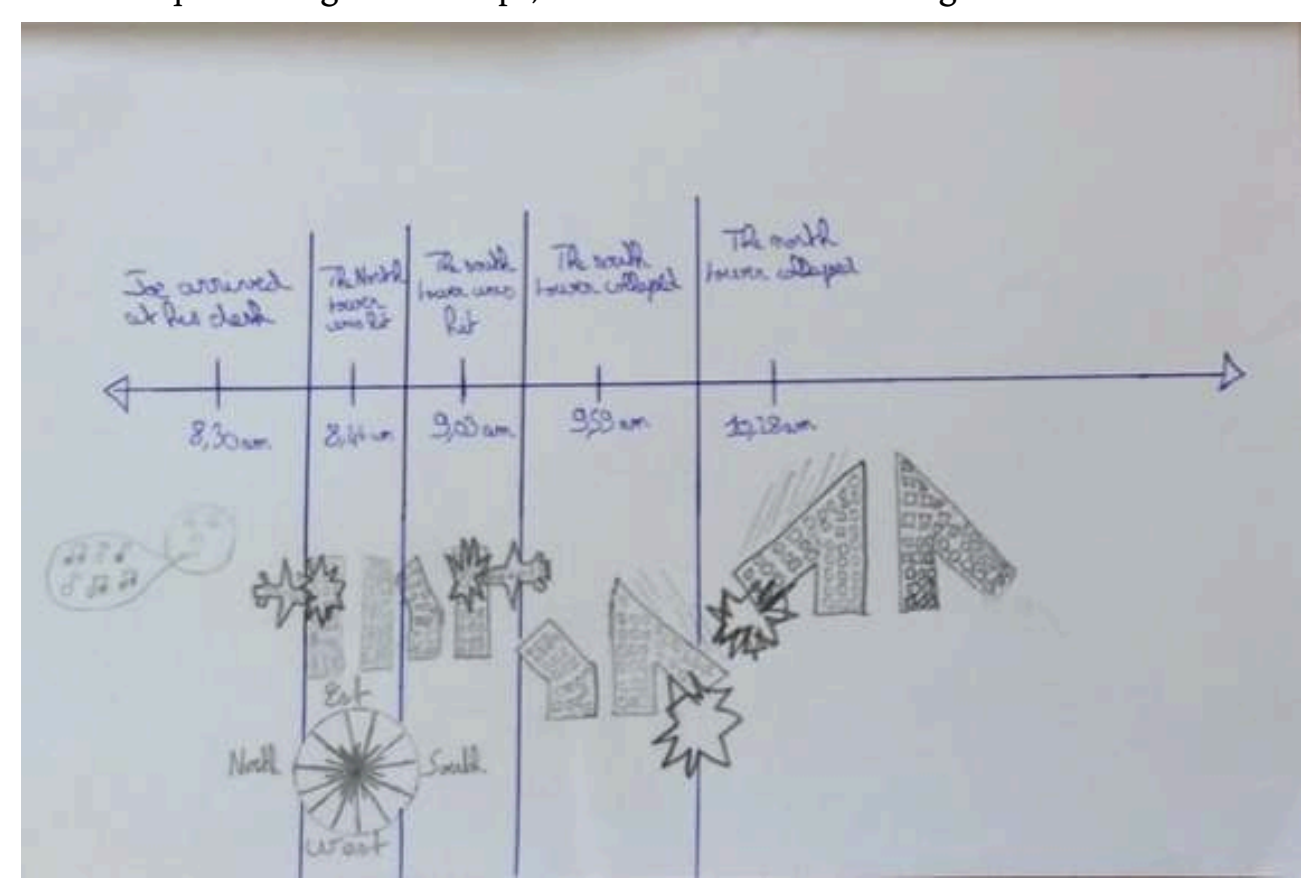

Représentation 5.

Lors de la phase de présentation à la classe, l'élève a argumenté son double fléchage par la préméditation des attentats, mettant en relation l'épisode des tours avec un 'avant'. Au-delà de cette explication très pragmatique, le double fléchage pose la question d'une part de l'orientation du temps-l'avenir vient-il à nous ou allons-nous vers lui- et d'autre part de la construction d'un point de vue subjectif à l'intérieur des moments créés. L'enseignante a donc pu en déduire qu'il y avait un terrain favorable à la conceptualisation de la représentation linguistique du temps, et notamment de l'utilisation conjointe et contrastée du prétérit, du present perfect et du pluperfect.

Et enfin, un élève a utilisé un repère orthonormé coordonnant la hauteur des tours au déroulement du temps : 


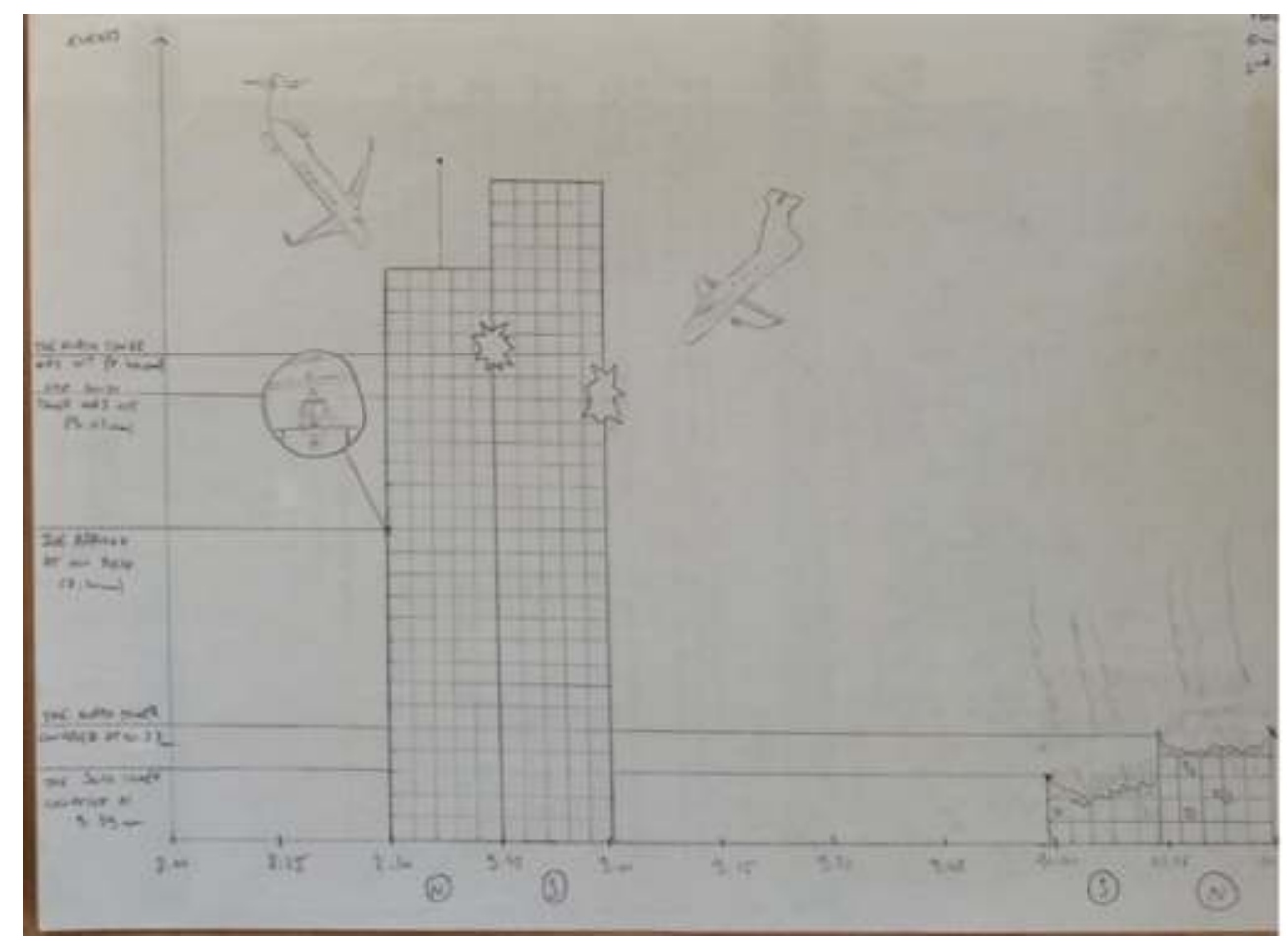

Document 6 .

La tentative d'abstraction dont témoigne cette production est intéressante : les phrases au prétérit sont étagées sur la gauche, axe de l'ordonnée, sans respect de l'ordre chronologique, ce qui selon la légende de ce repère orthonormé est logique puisqu'elles correspondent à la narration des événements qui ont conduit à la réduction de la hauteur des tours. L'abscisse représente la hauteur des tours en fonction des moments, l'ordonnée les événements. Il apparait donc que cet élève a voulu prendre en compte trois variables : hauteur des tours, succession des événements et temps chronologique. Or, événements et temps chronologique ne peuvent pas être posés sur deux axes différents qui détermineraient la hauteur des tours, comme si les différentes hauteurs de tours constituaient des éléments de la classe événements. Peut-être aurait-il fallu composer un repère à trois coordonnées, donc en trois dimensions: heures, événements, hauteur des tours. En outre, l'absence de production au présent ne permet pas d'être sûr que l'utilisation du prétérit est le résultat d'une bonne régulation, consciente ou inconsciente, de l'expression du passé en anglais.

C'est pourquoi cette activité individuelle de représentation a été suivie d'une explicitation orale qui a permis à chaque élève de s'approprier visuellement le concept même de chronologie à partir de l'expression d'une séquence d'événements. Cette séquentialité événementielle est essentielle à l'utilisation du prétérit simple en anglais, et a suscité une interaction orale dynamique et fructueuse fondée sur leurs propres perceptions de la représentation du temps, qui leur a permis de passer du système linguistique français au système anglais.

La possibilité qui a été donnée aux élèves de s'exprimer sur leur propre conception du rapport entre chronologie et référence au temps dans la langue et de discuter entre eux, suit le principe de Bange (1992) lorsqu'elle stipule que les reformulations proposées par l'enseignant en classe doivent confirmer un plan élaboré par l'élève afin de respecter son propre développement cognitif. C'est le respect de ce plan qui lui 
permet ensuite d'innover et de progresser selon une cohérence interne personnelle. Ce principe fait écho à la notion de la zone proximale de développement (ZPD), essentielle à la théorie de l'apprentissage de Vygotski (2003[1934] : 351).

\subsubsection{Troisième étape : Discussion autour d'une représentation du temps de l'événement} personnelles effectuées pour situer l'événement particulier du 11 septembre 2001 dans une histoire plus large des États-Unis ont été intégrées au résultat final.

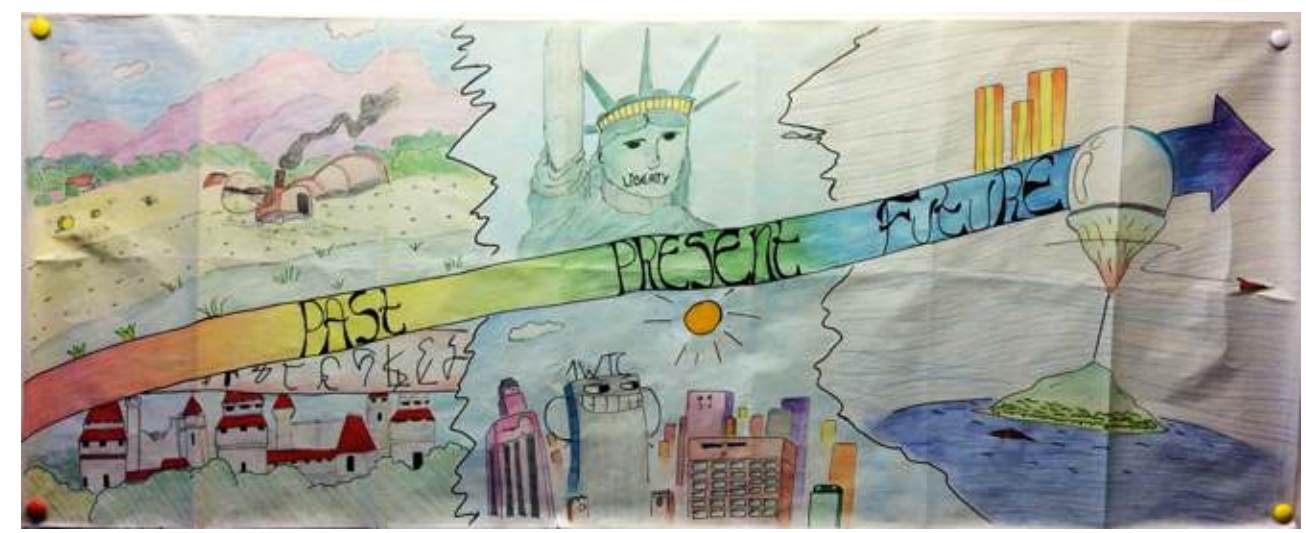

Représentation 7.

La réalisation de cette frise en grand format a été déléguée à 2 élèves plasticiens qui se sont porté volontaires pour la réaliser conjointement.

Les deux artistes ont présenté leur création oralement en classe pour obtenir la validation de leurs camarades. Ils ont décrit leur œuvre et justifié leurs choix graphiques, de couleurs et d'illustration soit en fonction de ce qui avait été discuté en classe précédemment soit en fonction d'une expérience scolaire commune de représentation symbolique de moments historiques. Si le choix d'un départ de flèche en 
retrait du bord de la feuille n'a pas été respecté, il s'agit d'un oubli, et non d'une intention, témoignant d'une fragilité de l'abstraction acquise antérieurement. Ce défaut a été résolu par le positionnement de la frise dans le cadre d'une autre feuille.

Si nous sommes encore loin de l'abstraction des appareils métalinguistiques sous forme de schémas topologiques (Leroux, 2018, Dufaye, 2008) pour représenter le temps, ce dessin, métaphore du temps, présente toutes les qualités pour être exploité collectivement en classe de seconde lors de phases d'explicitation de ce que représentent les désinences verbales en anglais.

\subsection{Quatrième étape : Exploitation de l'outil : de la production collective au support de médiation}

La production de l'outil de conceptualisation du temps ne pouvait pas constituer une fin en soi. Ainsi, cette métaphore du temps a-t-elle été systématiquement affichée au tableau et utilisée à chaque cours, suivant les besoins de la leçon, soit en phase de warm up, ou lors de l'introduction du past perfect pour que les élèves puissent visualiser, discuter puis matérialiser la succession d'actions passées et la relation qu'elles entretiennent entre elles, comme illustré sur la photographie suivante :

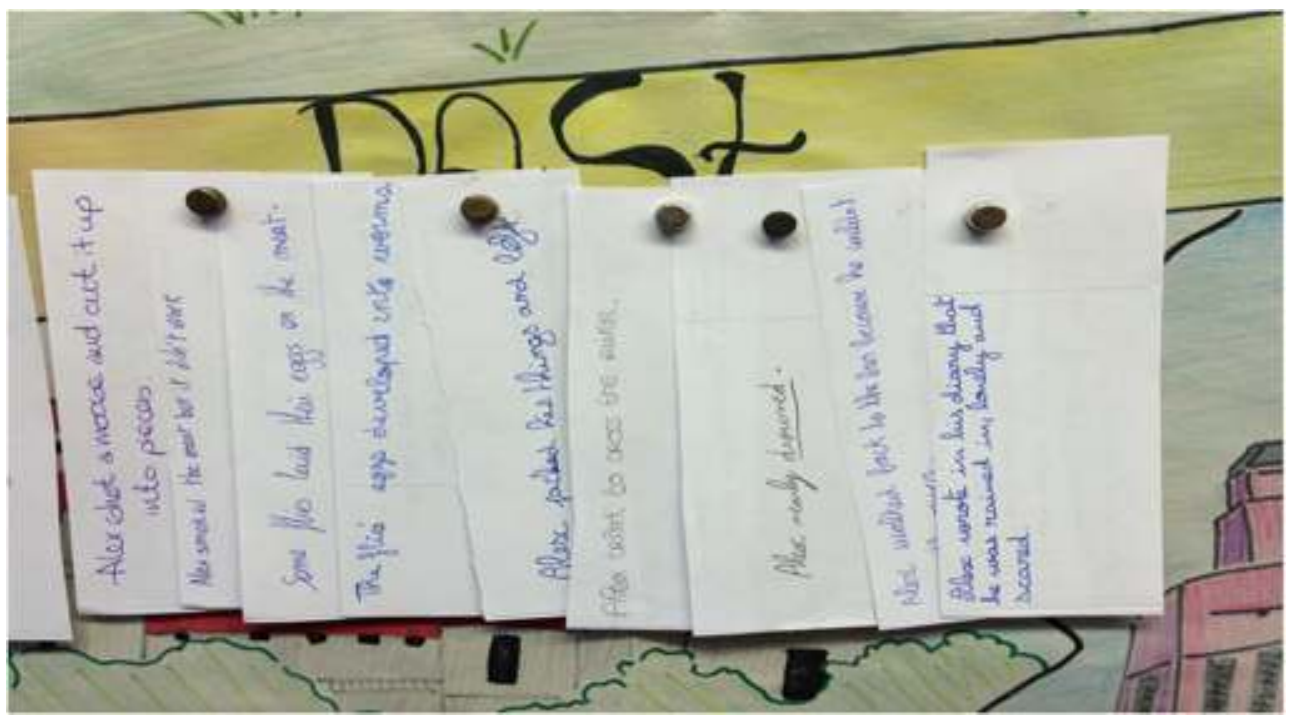

Représentation 8.

Sur chaque bande de papier est écrit un énoncé au prétérit, et chaque producteur est allé poser son énoncé sur la partie intitulée past de la frise chronologique. Ils ont ensuite eu à produire oralement des énoncés au pluperfect et devaient montrer quels énoncés étaient reliés entre eux. Cela leur a permis de reformuler le texte avec un support de réflexion métalinguistique.

Elle a ensuite servi de médiation à l'introduction-réactivation du present perfect lors d'une activité effectuée à propos des romans d'Agatha Christie à partir du document iconographique ci-dessous (illustration1) : 


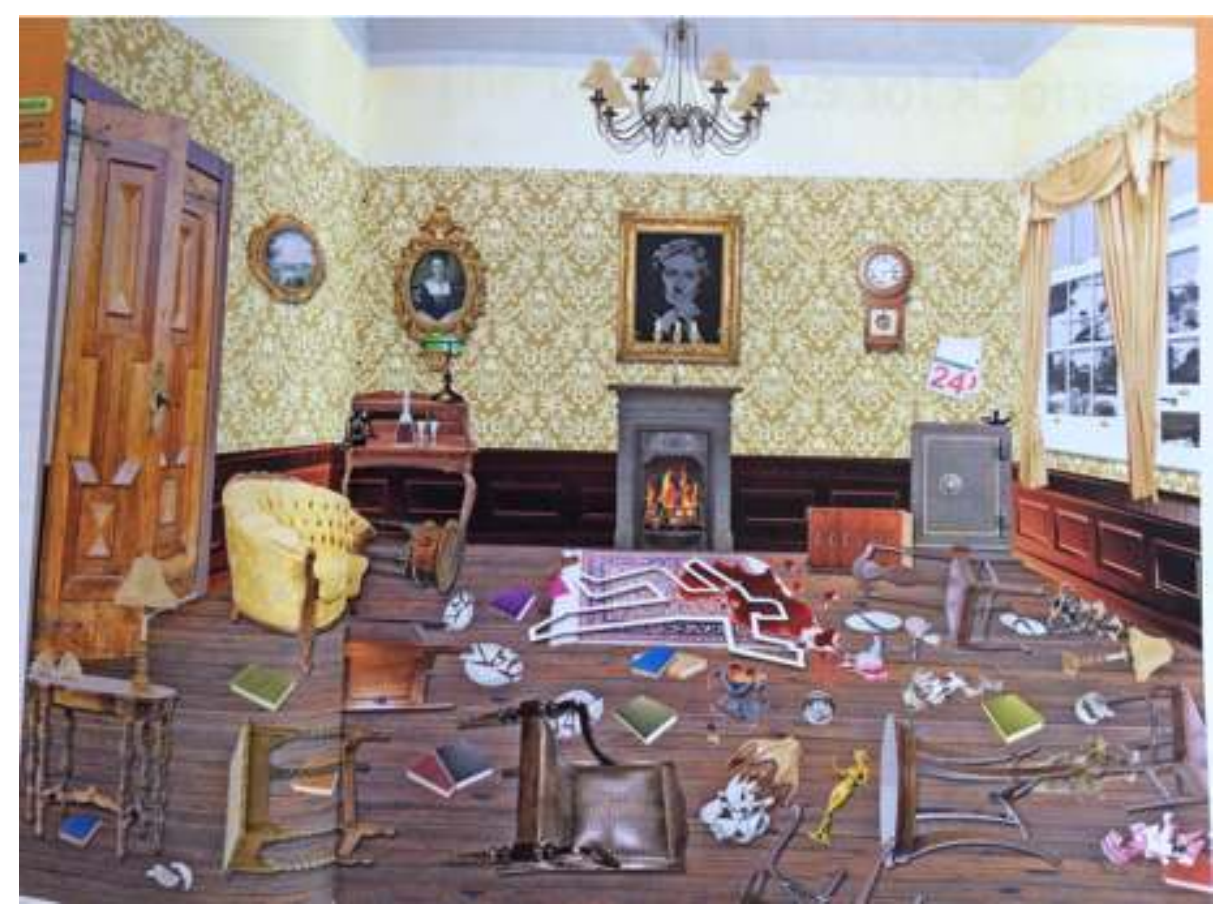

Illustration 1.

Les élèves ont dû imaginer ce qui venait juste de se passer, et donc situer leurs productions, en utilisant le même procédé qu'avec l'événement des Twin Towers, mais cette fois dans la partie présent de la frise chronologique.

Il apparait donc qu'en plus d'être le fruit d'une négociation collective impliquant les élèves autour d'un enjeu linguistique et langagier, la production matérielle est devenue support de médiation, puisque c'est autour de cet objet que se sont organisées les différentes discussions sur le rapport entre temps chronologique et temps grammatical, ainsi que la mise en forme de la référence aux événements en langue cible, l'anglais.

71 Ce support médiatique a évolué au fur et à mesure des différents moments de réflexion et les élèves ont été amenés à le peaufiner. Par exemple ils ont réfléchi à deux matérialisations différentes des deux prépositions for et since, selon la représentation qu'ils se faisaient du mode de fonctionnement de chacune.

S'inspirant de la représentation graphique de for élaborée et exposée par Leroux (2018) l'enseignante a proposé aux élèves un système de cases symbolisant la construction d'un intervalle de durée en une succession interne de moments identiques. Suite à discussion et négociation sur le sens construit par cette représentation, les élèves ont eu l'idée d'y inscrire le mot for (la lettre 0 étant représentée par un aimant permettant de fixer le tout sur la flèche chronologique). La pointe de flèche, qui peut sembler redondante avec celle de la flèche chronologique, est censée signifier selon eux jusqu'à aujourd'hui. Il est encore difficile pour des apprenants de quinze ans d'envisager le sens construit par for temporel indépendamment d'une chronologie, et l'enseignante, devant l'unanimité de la classe a préféré, là encore, respecter leur niveau de développement plutôt que de passer à côté de l'objectif visé. 


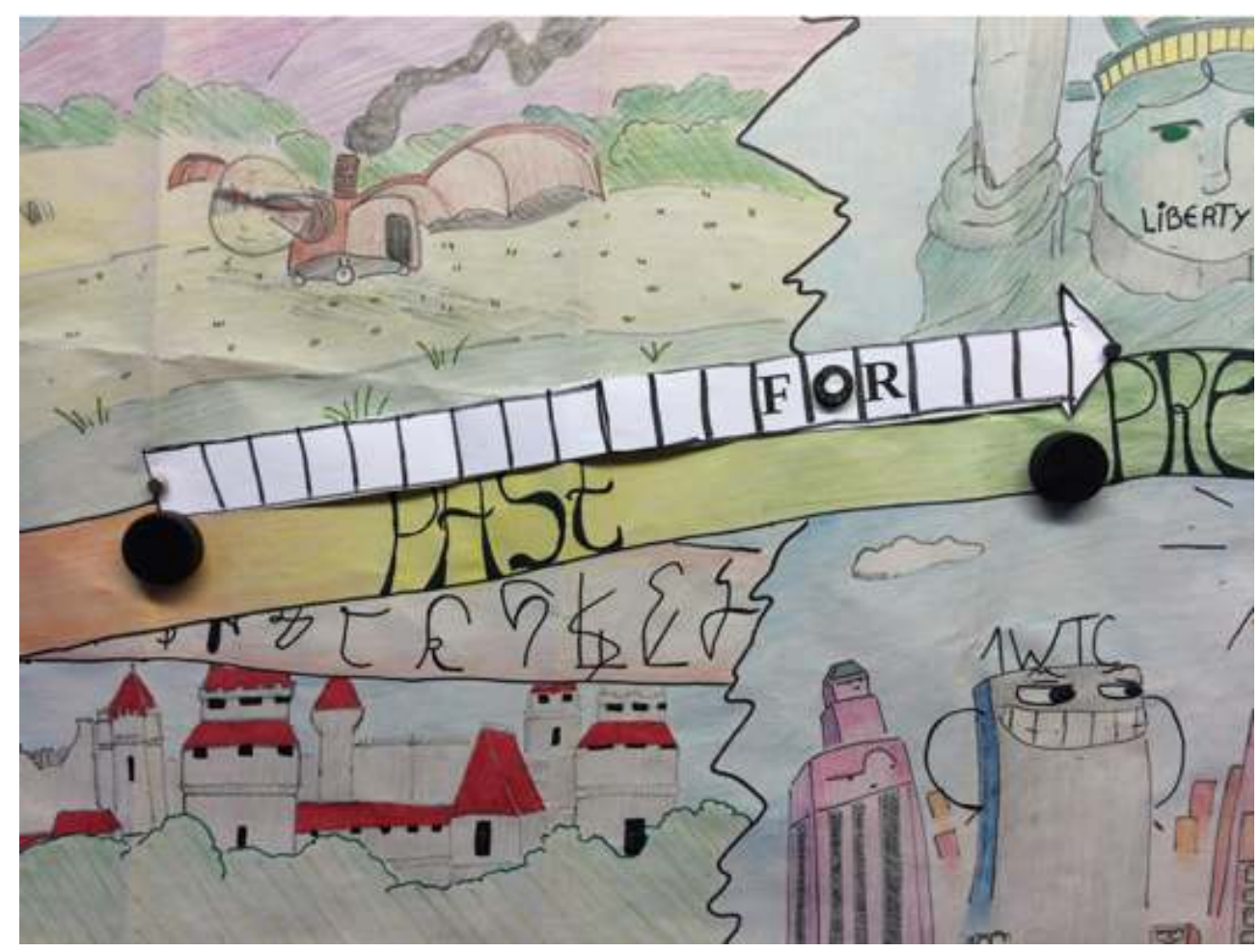

Représentation 9.

73 Néanmoins, le fait que for temporel ait été représenté par une flèche mobile laisse ouverte la possibilité future de repérer en bloc la durée ainsi construite par rapport à un moment en rupture par rapport au moment d'énonciation, et d'associer ainsi for + durée au prétérit.

La modélisation de since a également donné lieu à des discussions sur son mode d'intégration dans le dessin. Un élève a proposé d'utiliser une ficelle colorée mobile pour faire le lien entre ce moment passé début d'une durée et aujourd'hui, point final de l'intervalle. Il rendait donc visuelle la différence entre for et since: un intervalle plein contre un lien entre deux points dans le temps. Un autre élève a fait remarquer que l'aimant rond et noir utilisé pour marquer le début d'une durée pouvait être le point sur le $i$ du mot since que l'on pouvait écrire sur une étiquette. La classe a unanimement validé ces deux représentations. 


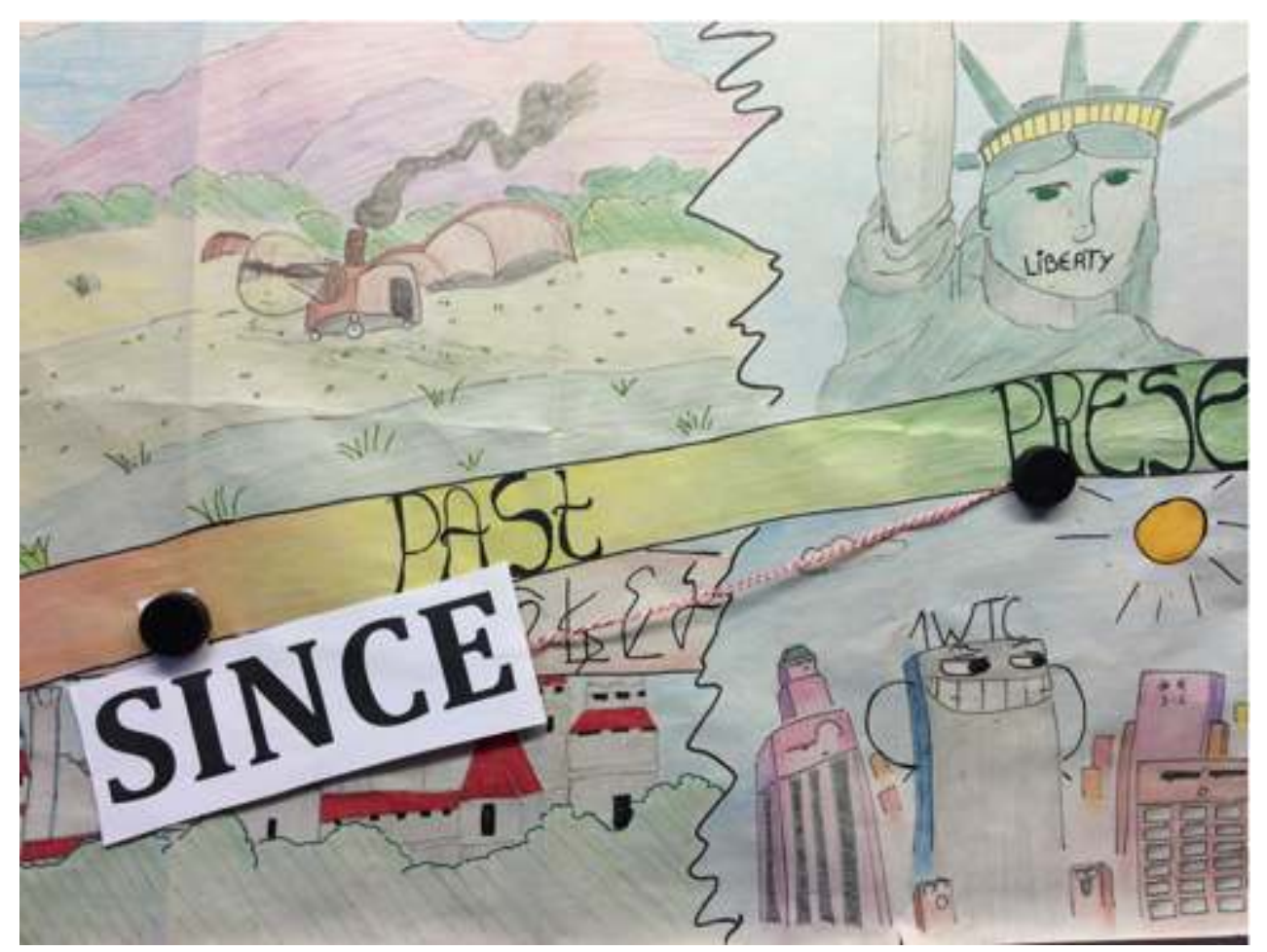

Représentation 10.

Par la suite, la flèche et ses accessoires ont été affichés systématiquement au tableau, afin que les élèves les aient sous les yeux à chaque cours et puissent y recourir en cas de désaccord ou de questionnement sur un élément temporel dans un texte ou au cours d'une activité d'expression. L'aspect inhabituel, voire ludique, de ces manipulations a d'ailleurs motivé les élèves à formuler des phrases requérant l'expression du passé ou de la durée.

\section{Résultats}

Cette expérience menée sur le terrain a donc concerné deux classes de seconde : une classe test, groupe expérimental, et une classe témoin, bénéficiaire d'un enseignement fondé sur une méthode actionnelle entrecoupée de quelques moments de focalisation sur les structures à l'œuvre dans le texte, à l'aide du précis de grammaire situé à la fin du manuel.

De la maîtrise initiale des compétences à l'évaluation de rétention au mois de mai, ou évaluation post-test, il apparaît que le groupe témoin a véritablement progressé, donc bénéficié de cette interprétation de l'approche à visée actionnelle ou de la pédagogie du projet.

78 Nous détaillons la progression ci-après.

\section{1 Évaluation initiale}

L'évaluation diagnostique initiale avait pour but, au-delà de la quantification ultérieure de la progression des élèves, d'évaluer la maîtrise, et le degré de maîtrise le cas échéant, de la compétence, et de repérer d'éventuelles disparités de niveau entre les 
deux groupes, qui pourraient fausser l'évaluation des procédés de remédiation. Elle a eu lieu fin novembre et a consisté en un exercice d'expression écrite semi-guidée portant sur les expériences de vie, avec une consigne précise, en français (afin de ne pas poser de problèmes de compréhension, ni d'influencer les productions), offrant un éventail d'exemples d'expériences et exigeant l'emploi de depuis. Entre trois et cinq phrases étaient demandées; les élèves disposaient de dix à quinze minutes pour la réalisation de l'exercice.

Compétence évaluée : savoir parler de ses expériences de vie.

Consigne : mentionnez un sport que vous pratiquez / un instrument de musique dont vous jouez / une langue que vous apprenez / quelqu'un que vous connaissez / le lieu où vous vivez... en précisant à chaque fois depuis combien de temps / depuis quand. Rédigez entre 3 et 5 phrases (une phrase par action).

Énoncé du test initial.

80 Cette évaluation a mis en évidence une différence de niveau notable entre les 2 groupes, prévisible puisqu'il s'agissait d'une Seconde Européenne SEA et d'une Seconde Nationale NAT.

Tableau 2.

\begin{tabular}{|l|l|l|l|}
\hline \multicolumn{3}{|l|}{ Bilan comparatif de la maîtrise de la compétence } \\
\hline \multicolumn{2}{|l|}{ Maîtrise du système temps-aspect } & \multicolumn{3}{l|}{ Maîtrise du système prépositionnel } \\
\hline Seconde européenne & Seconde nationale & Seconde européenne & Seconde nationale \\
\hline $4 / 25$ & $1 / 20$ & $20 / 25$ & $5 / 20$ \\
\hline
\end{tabular}

81 Sont présentés ci-dessous des exemples d'erreurs types rencontrées dans les deux catégories : maîtrise du système temps-aspect et maîtrise des prépositions, ainsi qu'une copie qui démontre une bonne maîtrise dans les deux domaines.

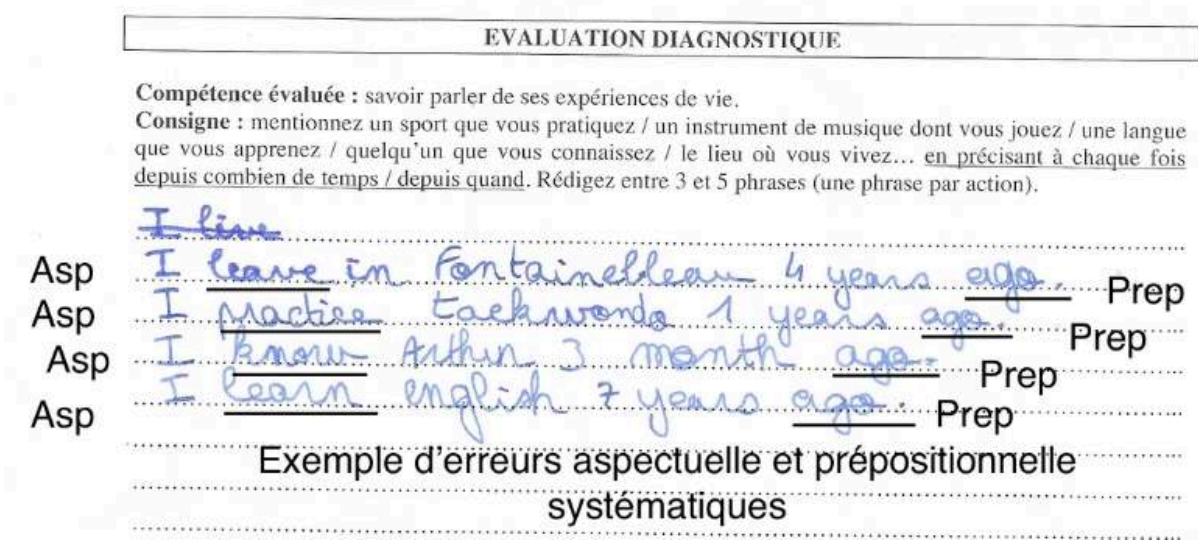

Évaluation 1

Groupe témoin : 
Compétence évaluée : savoir parler de ses expériences de vie.

Consigne : mentionnez un sport que vous pratiquez / un instrument de musique dont vous jouez / une langue que vous apprenez / quelqu'un que vous connaissez / le lieu où vous vivez... en précisant à chaque fois depuis combien de temps / depuis quand. Rédigez entre 3 et 5 phrases.

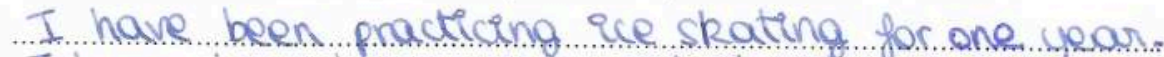
I have been learning spanish for thre veous I have peen liveng in Samereau since I was 10 . I have been playing the piano since last year...

\section{Exemple de bonne maîtrise de la compétence}

Évaluation 2 .

Tableau 3.

\begin{tabular}{|l|l|l|l|}
\hline \multicolumn{2}{|l|}{ Bilan comparatif de la maîtrise de la compétence après la phase expérimentale } \\
\hline \multicolumn{2}{|l|}{ Maîtrise du système temps-aspect } & \multicolumn{2}{l|}{ Maîtrise du système prépositionnel } \\
\hline Seconde européenne & Seconde nationale & Seconde européenne & Seconde nationale \\
\hline $14 / 25(+10)$ & $16 / 20(+15)$ & $23 / 25(+3)$ & $16 / 20(+11)$ \\
\hline
\end{tabular}

Présenter les résultats sur des cohortes aussi faibles en termes de pourcentages n'aurait pas été pertinent, et il n'est en aucun cas question ici de généraliser ces proportions à toute population scolarisée en seconde en France. Il apparaît néanmoins que la différence (chiffre entre parenthèses) entre le nombre d'élèves qui ne parvenaient pas à maîtriser les deux compétences linguistiques observées avant le processus de remédiation et le nombre de ceux qui y parviennent après, dans le groupe ayant bénéficié de l'approche expérimentale, est impressionnante. Les élèves du groupe test, en seconde européenne, qui ont travaillé sur les mêmes documents supports tout en s'appuyant sur le précis de grammaire du livre, ont également progressé et tiré profit du cours d'ALE, mais dans une moindre mesure.

La dernière évaluation, un post test effectué en fin d'année scolaire et prenant pour support une rédaction sur le sujet de leurs correspondants américains a montré de moins forts écarts. Les élèves du groupe témoin ont dû continuer à progresser et l'écart post expérience, certainement dû à la focalisation du groupe expérimental sur le fait de langue testé, s'est réduit. 
89 continuum d'évaluation :

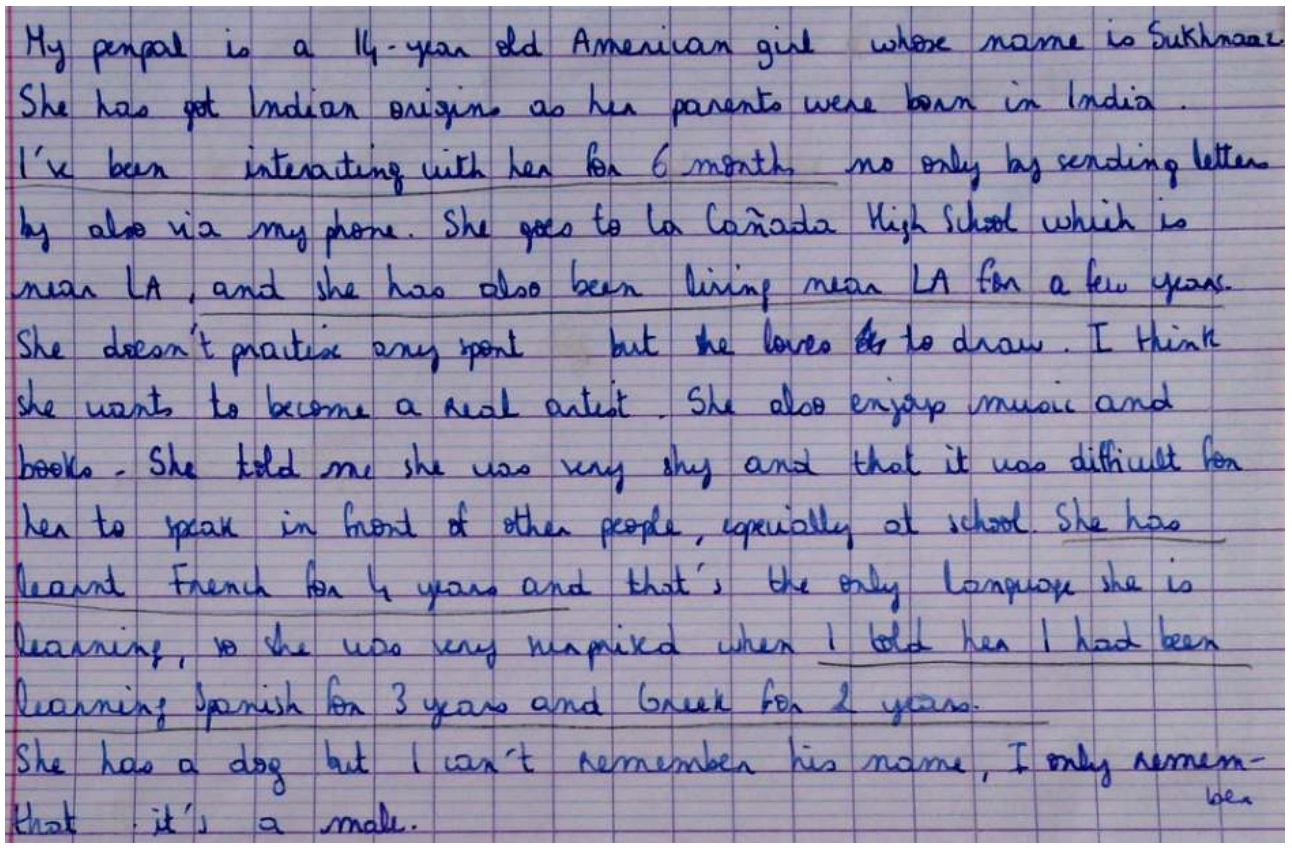

Évaluation 3.

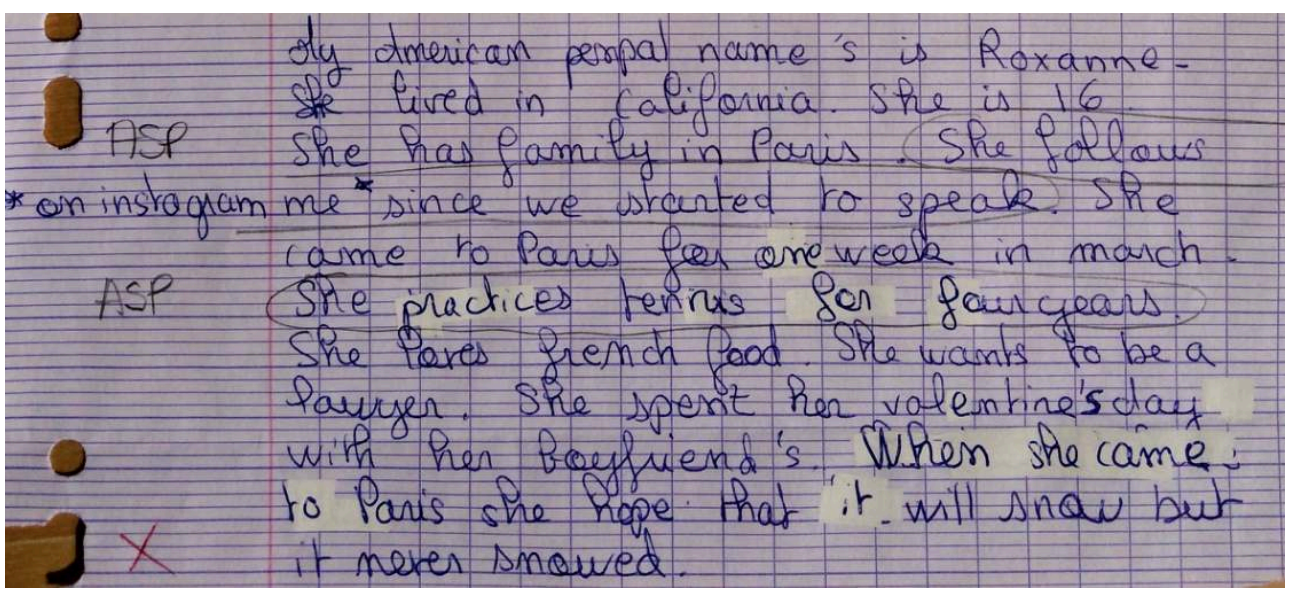

Évaluation 4.

Tableau 4.

\begin{tabular}{|l|l|l|l|l|l|}
\hline \multicolumn{5}{|l|}{ Bilan comparatif de la maîtrise des temps-aspects et prépositions en fin d'année } \\
\hline \multicolumn{2}{|l|}{ Acquis } & En cours d'acquisition & \multicolumn{2}{l|}{ Non acquis } \\
\hline $\begin{array}{l}\text { Seconde } \\
\text { européenne }\end{array}$ & $\begin{array}{l}\text { Seconde } \\
\text { nationale }\end{array}$ & $\begin{array}{l}\text { Seconde } \\
\text { européenne }\end{array}$ & $\begin{array}{l}\text { Seconde } \\
\text { nationale }\end{array}$ & $\begin{array}{l}\text { Seconde } \\
\text { européenne }\end{array}$ & $\begin{array}{l}\text { Seconde } \\
\text { nationale }\end{array}$ \\
\hline $16 / 25$ & $15 / 20$ & $6 / 25$ & $4 / 20$ & $3 / 25$ & $1 / 20$ \\
\hline
\end{tabular}


90 démontre la plus grande efficacité du procédé expérimental sur le long terme. En effet, on constate un taux de fixation, c'est-à-dire d'élèves ayant maintenu leur maîtrise de la compétence, supérieur pour le groupe test.

Néanmoins, force est de constater une moins forte régression des élèves du groupe test, c'est-à-dire d'élèves passés d'une bonne maîtrise à une maîtrise partielle, que du groupe témoin. Enfin, un seul élève du groupe test n'a jamais acquis la compétence, contre trois dans le groupe témoin.

Le bilan du processus expérimental est donc, de notre point de vue d'enseignante, globalement très positif: non seulement le groupe test a bien mieux progressé que le groupe témoin, mais son degré de maîtrise de la compétence est nettement supérieur, alors même qu'au départ le groupe témoin avait obtenu de meilleurs résultats à l'évaluation diagnostique.

93 On peut donc dire que dans le cadre de cette expérience, pour l'apprentissage du système des temps et des aspects en cours d'ALE, la construction d'un projet de séquence autour d'un fait de langue s'est révélée pédagogiquement efficace.

\section{Respect du cahier des charges de l'enseignante vis à vis de l'institution}

94 Si nous reprenons le texte cité en ouverture de cet article, nous pouvons montrer que les points surlignés ont été respectés :

En classe, l'enseignant privilégie la mise en place de projets autour de scénarios pédagogiques qui articulent les entraînements, les contextualisant et aboutissent progressivement à des productions écrites ou orales (...). L'enseignant s'attache à mettre l'élève en action et l'encourage à prendre des initiatives à travers les activités proposées.

- Mise en place de projets : il s'agissait de l'élaboration d'un outil métalinguistique pour conceptualiser la construction linguistique du temps chronologique en anglais, de façon collective et négociée ;

- Contextualisation des entrainements : travail sur des documents support authentiques, ouvrant à la discussion et à l'utilisation du dispositif construit collectivement, et expression sur des situations réelles qui concernent les élèves, comme la relation à leur correspondant américain

- Élaboration progressive de productions écrites ou orales : ce dispositif a aidé les élèves à produire à l'oral et à l'écrit et à améliorer la qualité linguistique de leurs productions.

- Encouragement à prendre des initiatives : représentations additionnelles de for, et de since, et évolution du schéma, encouragées par l'enseignante.

95 Il apparaît donc que ce travail a été effectué dans le respect du contrat qui lie l'enseignante à son employeur, l'Éducation Nationale.

\section{Conclusion}

Les résultats de cette expérience ne peuvent bien entendu pas être généralisés à tout élève de classe de seconde scolarisé en France. Malgré tout, le bilan dressé en fin d'année scolaire est encourageant. 
97 Les résultats des évaluations effectuées à distance de la séquence montrent que redonner à la langue sa place d'objet d'étude au sein du cours de LVE en milieu institutionnel peut se révéler efficace à plusieurs niveaux. Tout d'abord, la dissipation du malentendu sur l'objectif du cours de langue que peut provoquer l'approche à visée actionnelle incite les élèves, même en difficulté, à prendre part aux échanges. Ensuite, la création d'un outil négocié collectivement et exploitable en classe donne une légitimité à l'interlangue de l'élève en même temps qu'elle lui montre un chemin pour la progression. Enfin, la réflexion sur le rapport en LS et LVE que l'utilisation de l'outil remet constamment en œuvre incite les élèves à s'exprimer et à conceptualiser à partir des représentations langagières.

Comme mis en évidence dans notre quatrième partie, le cahier des charges imposé par les IO a été respecté, en même temps que les élèves ont eu le loisir d'exercer leur réflexion pour construire leur propre compréhension du système linguistique de l'anglais. En cela nous avons montré par cet exemple que les principes de psycholinguistique de Chini étaient applicables et efficaces.

99 Malgré tout, cette expérience, menée progressivement au fur et à mesure du premier semestre montre quelques faiblesses et points d'amélioration. En effet, la négociation de la construction de l'outil métalinguistique s'est faite en français, parce que l'enseignante voulait que les élèves se sentent libres de discuter avec précision de leur propre conception du temps. En cela, le projet exposé ici relève peut-être plus du 'méta-projet' pour le cours de LVE que du projet en cours de LVE. Une négociation en tout anglais, même si se reposerait alors le problème de l'utilisation de la LVE pour étudier la LVE, pourrait faire l'objet d'une prochaine expérience. D'autre part, les tests d'évaluation de la rétention de la forme linguistique étudiée ont été effectués à l'écrit ; il faudrait envisager un test d'impact de ce type de séquence sur la progression de la compétence langagière de communication à l'oral.

Il reste que ce projet renforce la collaboration sur la recherche entre l'enseignement supérieur et l'enseignement secondaire, et amène l'enseignant du secondaire à se questionner sur l'objet de son propre projet d'enseignement et l'enseignant du supérieur à envisager les applications pratiques de la recherche scientifique en psycholinguistique. Il s'agit alors de dépasser une conception de l'enseignement des LVE en milieu institutionnel comme l'application de préconisations, pour mettre en œuvre une pédagogie fondée sur une réflexion didactique et psycholinguistique, à la fois scientifique et personnelle.

\section{BIBLIOGRAPHIE}

Audin, L., et Institut National de Recherche Pédagogique, 2005, Enseigner l'anglais de l'école au collège : comment aborder les principaux obstacles à l'apprentissage, Hatier, Paris.

BAILLY, D., 1984, Éléments de didactique des langues : l'activité conceptuelle en classe d'anglaisdeuxième partie : une étude de corpus, Les Langues Modernes, APLV, Lyon. 
BANGE, P., 1992, «À propos de la communication et de l'apprentissage de L2 (notamment dans ses formes institutionnelles) », Acquisition et interaction en langue étrangère [En ligne], 1 | 1992, <http://journals.openedition.org/aile/4875>.

BRONCKART, J.-P., 1994, « Action, langage et discours. Les fondements d'une psychologie du langage ", Bulletin Suisse de Linguistique Appliquée, n59, Neuchâtel, p. 7-64.

BROSSARD, M., 1994, «Quelques réflexions sur : Activités métalinguistiques et situations scolaires », Repères. Recherches en didactique du français langue maternelle 9 (1), p. 29-36. <https://doi.org/10.3406/reper.1994.2107>

CHINI, D., 2009, « Linguistique et didactique : où en est-on? Quelle place pour une approche conceptualisante de la construction de la langue dans la perspective actionnelle ? ", Recherches en Didactique des Langues et des Cultures 6, $\mathrm{n}^{\circ} 2$, <http://journals.openedition.org/rdlc/1958>

Culioli, A., 1999, Pour une linguistique de l'énonciation, Tome 2, Ophrys, Paris.

DUFAYE, L., 2009, Théorie des opérations énonciatives et modélisation : cheminement d'une réflexion linguistique, Col. L'homme dans la Langue, Ophrys, Paris.

ElLIS, R., 2018, Reflections on Task-Based Language Teaching, Second Language Acquisition 125, Blue Ridge Summit, PA, Multilingual Matters, Bristol.

GOMBERT, J.-É., 1996, « Activités métalinguistiques et acquisition d'une langue », AILE, 8

(Décembre), p. 41-55.

LEROUX, A., 2018, La construction linguistique de la durée en anglais et en français, Col. Gramm'R, Peter Lang, Bruxelles.

LEROUX, A., PLESSIS, V., à paraître, « De l'épilinguistique au métalinguistique en cours d'Anglais Langue Étrangère (ALE) au lycée : un réel projet pour le cours de langue » dans [titre noncommuniqué] Dufaye, L., Gournay, L. (éds), Lambert-Lucas, Limoges.

TARDIF, J., 1992, Pour un enseignement stratégique : l'apport de la psychologie cognitive. Col. Théories et Pratiques d'Enseignement, Éditions Logiques, Montréal.

TRÉVISE, A., 1994, « Représentations métalinguistiques des apprenants, des enseignants et des linguistes : un défi pour la didactique », Bulletin Suisse de Linguistique Appliquée, $n^{\circ} 59$, Neuchâtel, p. 171-190.

VyGotski, L., 2003, Pensée et langage, La Dispute, Paris.

Manuel d'anglais Langue Étrangère :

Fort-Couderc M., Guary F., Manescau G., Persec S., Tripodi S., 2010, New Bridges Seconde, Paris, Nathan.

\section{RÉSUMÉS}

Cet article tente d'apporter une solution au paradoxe de l'enseignement des langues en milieu institutionnel en France généré par les injonctions contradictoires à destination des enseignants. Les Instructions Officielles françaises soulignent non seulement la nécessité de l'exposition fréquente à la langue mais aussi l'importance d'amorcer une réflexion sur son fonctionnement pour développer autonomie et maîtrise, sans oublier la mise en œuvre d'une pédagogie de projet afin de favoriser les échanges en classe. Les horaires dédiés à l'enseignement des langues ne donnent pas le loisir d'appliquer toutes ces consignes à la fois. L'expérience présentée et analysée 
dans cet article, est fondée sur le principe de l'efficacité d'une séquence qui prend pour tâche collective et finale la construction d'un outil métalinguistique pour décrire un fait de langue. Partant du constat, suite à évaluation diagnostique, que le système aspectuo-temporel de l'anglais constituait une difficulté conceptuelle particulière pour les apprenants francophones, une classe de 2 de a été engagée sur le chemin de l'élaboration d'un outil métalinguistique qui permette à la fois sa description et la remédiation des erreurs. En parallèle, un groupe classe témoin permet de jauger les progrès réalisés dans la classe test. Le versant linguistique de l'analyse prend pour cadre la Théorie des Opérations Prédicatives et Enonciatives, et s'appuie, entre autres, sur les travaux de Berthonneau (1992), de Franckel (1989) et de Trévise (1990). Les résultats montrent que le temps dédié à la réflexion est du temps gagné sur l'apprentissage et sur la compétence à communiquer.

This article focuses on the difficulties that the French secondary school teachers encounter when it comes to respecting all the injunctions which govern their profession. The national curriculum states that the pupils should be exposed as often as possible to authentic occurrences of language, exercise their grammatical awareness, take part in conversations with their peers, and elaborate a final collective task, which is not necessarily linguistic. But the foreign language schedule in French schools compels the teachers to make a selection among all those objectives. The experiment presented and analysed here shows that a foreign language teaching sequence in which the final task aims at the elaboration of a metalinguistic tool might gain in efficiency, while respecting the national curriculum.

\section{INDEX}

Keywords : EFL didactics, grammar, conceptualization, secondary school instruction, tense and aspect.

Mots-clés : didactique de l'ALE, conceptualisation, grammaire, enseignement secondaire, temps et aspect.

\section{AUTEURS}

\section{AGNÈS LEROUX}

Université de Paris Nanterre, équipe CREA, lab. GReG, EA 370

\section{VIRGINIE PLESSIS}

Prof. Agrégée en lycée, Académie de Créteil 